\title{
An all fiber based Talbot self-imaging mirror device for phase-locking of a multi-fiber laser
}

\author{
R. Zhou \\ Electro-Optics Program, University of Dayton, 300 College Park, Dayton, OH 45469, USA \\ Q. Zhan \\ Electro-Optics Program, University of Dayton, 300 College Park, Dayton, OH 45469, USA \\ P. E. Powers \\ Electro-Optics Program, University of Dayton, 300 College Park, Dayton, OH 45469, USA \\ Physics Department, University of Dayton, 300 College Park, Dayton, OH 45469, USA \\ B. Ibarra-Escamilla \\ Instituto Nacional de Astrofisica, Opticay Electronica Optics Department, Luis Enrique Erro No. 1, \\ Puebla, Pue, 72000, Mexico \\ J. W. Haus \\ jwhaus@udayton.edu \\ Electro-Optics Program, University of Dayton, 300 College Park, Dayton, OH 45469, USA
}

By propagating beamlets from a periodically placed array of single-mode fiber amplifiers in a large mode-are (LMA) fiber, we find that a self-image of the initial beamlets is formed at certain distances in the LMA fiber. A Talbot mirror fiber device (TMFD) based on this property is proposed for phase-locking of a multi-fiber laser. For this laser system, we investigate how the LMA fiber length variation; the fiber amplifier phase variation, amplitude variation, and displacement affect the self-image qualities and the coupling efficiency.

[DOI: http://dx.doi.org/10.2971/jeos.2012.12012]

Keywords: talbot and self-imaging effects, fiber laser, phase-locking

\section{INTRODUCTION}

Fiber lasers are a promising candidate for developing high power lasers [1, 2]. One reason is that optical fibers have simple geometries and a small volume which makes alignment relatively easy and the laser cavity compact. Also, optical fibers have an inherently high surface area to active volume ratio which allows for efficient cooling. However, in a single fiber the output power is limited by nonlinear effects such as stimulated Raman scattering and stimulated Brillouin scattering [3, 4]. Using large mode-area (LMA) fibers designed with single modes can reduce nonlinear effects by spreading the power over a wider area; single-mode output power up to $10 \mathrm{KW}$ has been demonstrated by IPG Photonics [5]. However, due to the mode confinement limitations in conventional optical fibers, maintaining lasing with single-mode output in an LMA fiber is difficult.

Another strategy for high power fiber lasers was proposed by Siegman, who published a complete description of gainguiding in slab-waveguides and optical fibers, which can maintain a single mode profile with core size up to several hundred microns [6]. Lasing in gain-guided, index antiguided (GG+IAG) fiber with single mode output has been demonstrated experimentally recently by several researchers [7]-[9]. With fiber lasers in mind, recent papers have studied gain-saturation effects and self-focusing in a gain guided waveguide [10]-[12]. However, the pump technique for this fiber needs to be improved, and the pumping efficiency is extremely low.
At the same time, phase-locked fiber laser designs have been theoretically and experimentally demonstrated to be a good candidate for the development of high power fiber lasers $[13,14]$. More importantly, self-organized coherence in fiber laser arrays has been discovered and studied recently [15][17], which eliminates the need for active feedback control for the fiber length or polarization.

In a compact design Li et al demonstrated the use of a passive fiber as a Talbot mirror [18, 19], using self-imaging in multimode optical fibers [20]. Their fiber laser cavity uses multicore fiber where cross talk can occur. The use of a multi-core fiber also limited the pump coupling efficiency.

In this paper, we propose an all fiber-based compact phaselocked multi-fiber laser design using the Talbot self-imaging effect. Similar to Ref [18, 19], we use a Talbot mirror fiber device (TMFD) which serves as an imaging resonator. The TMFD is a large mode area fiber (LMA) of a certain length with a partial reflector fabricated at one end. The LMA fiber length is determined by numerically solving the beam propagation in the fiber. Numerical simulation results display phased array results for 4 and 8 fibers periodically placed in regular polygonal geometry. Also the simulation is performed with an additional fiber in the center for 4 and 8 fibers cases and the power coupling efficiency is significantly improved. In addition, we have studied the self-imaging instability by changing the LMA fiber length; varying the phase, amplitude, and position among the input SM fibers. 


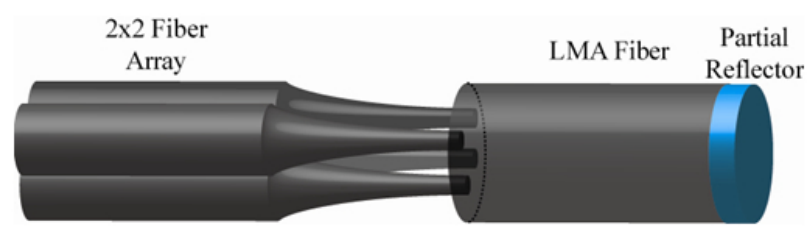

FIG. 1 Phase-locking fiber laser design showing the TMFD end section. A $2 \times 2$ fiber amplifier array is coupled into a $2 \times 2$ set of SM fibers which are fused onto a LMA fiber in a regular rectangular geometry. A partial reflector acts as the output coupler is fabricated on the opposite end of the LMA fiber. Also, there could be fiber Bragg gratings which $100 \%$ pass the pump and $100 \%$ reflect the signal at the input end of each fiber amplifier to form the laser cavity.

\section{TALBOT MIRROR FIBER DEVICE (TMFD) CONCEPT}

The TMFD is a very compact device which consists of a LMA fiber and a partial reflector; it is illustrated in Figure 1. This fiber device is the key component of the fiber laser cavity design. As illustrated, initial beamlets delivered by a fiber array are coupled into the LMA fiber After its forward propagation in the LMA fiber the beam is partially reflected and back propagates to the input plane. After one round trip propagation in the LMA fiber, the beam self-images at the input plane, provided the input beams are properly phased. The TMFD reinforces a phased array since the feedback is maximum for a reimaged profile, which corresponds to the properly phased array. The LMA fiber length is carefully selected to ensure the self-imaging recurs at the input plane to maximize the power coupling efficiency between the reflected beam and the fiber array. In this paper, the LMA length is half of the selfimaging distance. However, a full self-imaging distance can also be used. We design the TMFD geometry through numerical simulations of beam propagation in the LMA fiber, and we limited the propagation length to be within $20 \mathrm{~mm}$ which is enough for finding a self-imaging distance.

\section{PHASE-LOCKED MULTI-FIBER LASER DESIGN}

For the phase-locked multi-fiber laser design illustration, we use a $2 \times 2$ fibers array as an example. In Figure 1, a $2 \times 2$ fiber amplifier array is coupled into a $2 \times 2$ set of multi-mode (MM) fibers which is then tapered down to SM fibers array. The tapering can be done with a Vytran GPX 3400, see Ref [21]. After that, the SM fibers array is coupled into the LMA fiber. A partial reflector, which is also the output mirror, is fabricated at the opposite LMA fiber end. By carefully choosing the length of the LMA fiber the reflected light forms a self-image at the input plane and couples back into the SM fibers array. We note that the tolerance on the length of the LMA fiber is relaxed somewhat if the fiber amplifiers have modest tunability. The coupling efficiency due to the re-imaging is crucial for operating the laser system. There are also mirrors at the other end of the fiber amplifiers, which could be fiber Bragg grating that pass $100 \%$ of the pump while reflecting $100 \%$ of the signal. The end mirrors and the TMFD form a resonator. The cavity is self-organized to lase using a low loss mode by adjusting the phases and amplitudes in each injected amplifier [15].

\section{NUMERICAL SIMULATIONS OF SELFIMAGING IN LMA FIBER}

In this section the self-imaging effect is studied for four different SM fibers arrays, including: four periodically placed fibers on the vertices of a square, four periodically placed fibers plus one fiber in the center, eight periodically placed fibers on the vertices of a regular octagon, and eight periodically placed fibers plus one fiber in the center. We assume Gaussian signal beams with flat phase profiles coming from each of the SM fibers (Thorlab's SMF-28 fiber) with a mode field diameter of $10.4 \mu \mathrm{m}$, and a signal wavelength of $1.55 \mu \mathrm{m}$. Also, we assume no phase or power variations between each beam. The LMA fiber (Thorlab's BFL37-200 fiber) that is used has a $200 \mu \mathrm{m}$ core diameter, and the core and cladding index are estimated to be 1.506 and 1.460, respectively. For the model, we assume the cladding is infinite. The reflector fabricated on the LMA fiber end has $50 \%$ power reflection at $1.55 \mu \mathrm{m}$. We first show the case of four periodically placed fibers coupled to the LMA fiber. The SM fibers are fused to the LMA fiber in a square array with side length of $60 \mu \mathrm{m}$. In order to find the optimum self-imaging distance, we simulate the beam propagation in the LMA using a standard Fast-FourierTransform (FFT) method, see video, say for Figure 2(a). With the video as a guide, we numerically find that a self-imaging distance is about $11.28 \mathrm{~mm}$ where the maximum power coupling efficiency into the SM fibers happens. The power coupling efficiency is calculated through Ref [22] page 271-274, and the maximum power coupling efficiency is found to be about $16 \%$, and the total efficiency, which is defined as the ratio of the coupled power back into the SM fibers to the initial input power, is about $6 \%$. The total efficiency includes the $50 \%$ power loss from output coupler, and the loss caused by the radiation modes in the fiber core. The videos for the cases of $4+1$, 8 and $8+1$ beamlet cases are linked in Figure 2.

In Figure 3, we show the simulation results with LMA length of $5.64 \mathrm{~mm}$, which is half of the reimaging distance. Note that, we only look at the beams in the fiber within 50 microns radius from the center, where most of the beam is concentrated. Figures 3(a) and 3(e) are the input field intensity and phase distribution, Figures (b) and (f) are the self-imaged (i.e. round trip) field intensity and phase distribution at the input plane, and Figures 3(c) and (g) are the field intensity and phase distribution at the partial reflector, respectively. The output far-field intensity and phase distributions are plotted in Figures 3(d) and $3(\mathrm{~h})$, respectively. It can be seen that most of the power is concentrated in the center. If an adaptive element is placed after the output coupler to correct the phase, such as a Stimulated Brillouin Scattering (SBS) conjugate mirror [23], the far field beam quality will be improved.

For the four periodically placed fibers array plus one fiber in the center case, four SM fibers are fused to the LMA fiber in a square array with side length of $60 \mu \mathrm{m}$, and an additional identical fiber is fused to the center of the LMA fiber. The beam propagation in the LMA is shown in a video, see Figure 2(b). When the LMA fiber length is $7.29 \mathrm{~mm}$ corresponding to a reimaging distance of $14.58 \mathrm{~mm}$, we find the maximum field coupling efficiency into the SM fibers. We note that another self-image also occurs around $11.28 \mathrm{~mm}$, but the coupling ef- 


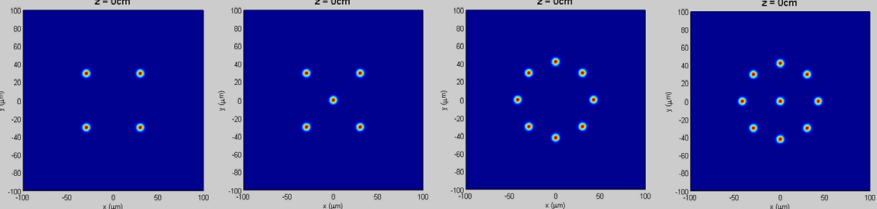

FIG. 2 Beam propagation in LMA fiber. (a) is the propagation for 4 fibers array case (Movie-1), (b) is the propagation for $4^{+1}$ fibers array (Movie-2), (c) is the propagation for 8 fibers array case (Movie-3), and (d) is the propagation for $8+1$ fibers array case (Movie-4).
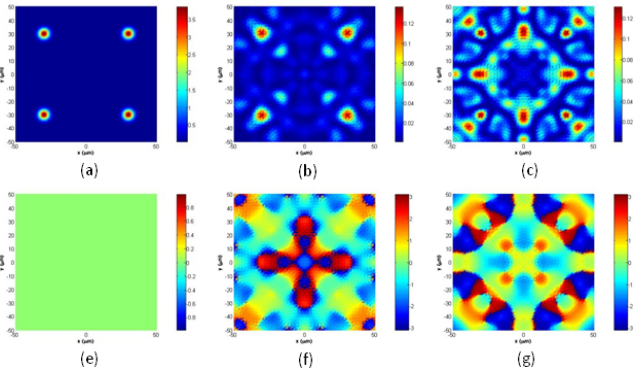

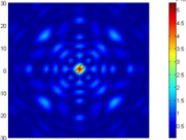

(d)

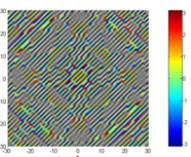

FIG. 3 Simulation of self-imaging for 4 periodically placed fibers array in a $5.64 \mathrm{~mm}$ long LMA fiber. The top row is the intensity profile and the bottom row is the phase profile. The propagation distances are paired together: $(a, e) \circ \mathrm{mm},(b, f) 11.28 \mathrm{~mm}$ (round trip propagation image at the input plane), $(c, g) 5.64 \mathrm{~mm}$, and $(\mathrm{d}, \mathrm{h})$ is the far field diffraction pattern.

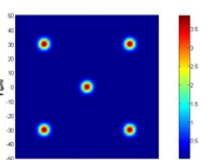

(a)

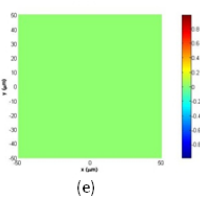

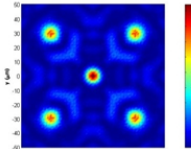

(b)

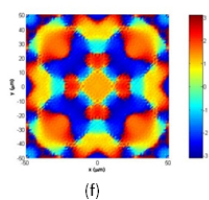

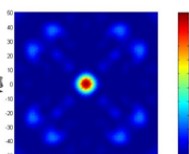

(c)

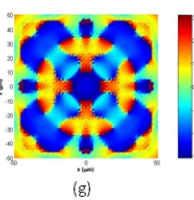

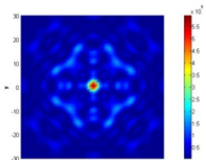

(d)

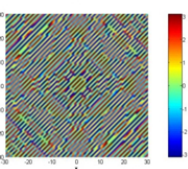

(h)
FIG. 4 Simulation of self-imaging for $4^{+1}$ periodically placed fibers array in a $7.29 \mathrm{~mm}$ Iong LMA fiber. The top row is the intensity profile and the bottom row is the phase profile. The propagation distances are paired together: $(a, e)$ omm, $(b, f) 14.58 \mathrm{~mm}$ (round trip propagation image at the input plane), $(c, g) 7.29 \mathrm{~mm}$, and $(\mathrm{d}, \mathrm{h})$ is the far field diffraction pattern.

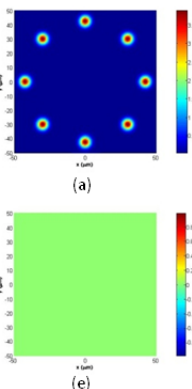

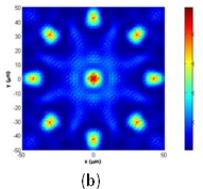

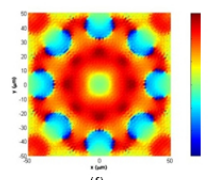

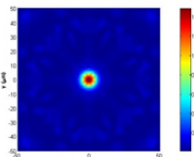

$($ c)

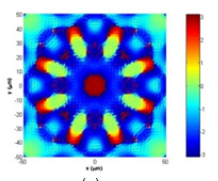

(g)
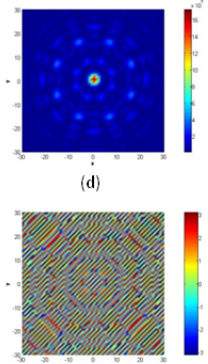

(h)
FIG. 5 Simulation of self-imaging for 8 periodically placed fibers array in a $7.42 \mathrm{~mm}$ Iong LMA fiber. The top row is the intensity profile and the bottom row is the phase profile. The propagation distances are paired together: $(a, e) \circ \mathrm{mm},(b, f) 14.84 \mathrm{~mm}$ (round trip propagation image at the input plane), $(c, g) 7.42 \mathrm{~mm}$, and $(\mathrm{d}, \mathrm{h})$ is the far field diffraction pattern.

ficiency is much lower through numerical calculation. This
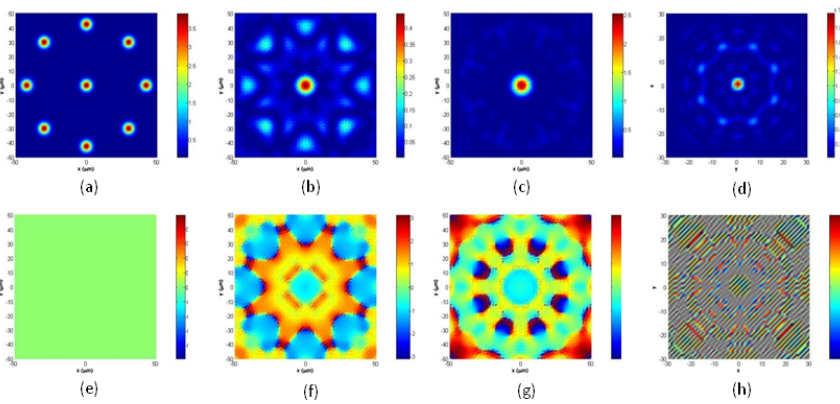

(d)
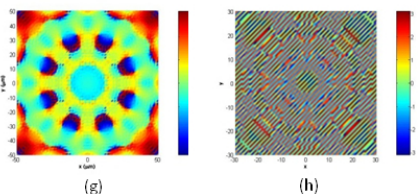

(h)

FIG. 6 Simulation of self-imaging for $8+1$ periodically placed fibers array in a $7.3 \mathrm{~mm}$ long LMA fiber. The top row is the intensity profile and the bottom row is the phase profile. The propagation distances are paired together: $(a, e) 0 \mathrm{~mm},(b, f) 14.60 \mathrm{~mm}$, $(c, g) 7.30 \mathrm{~mm}$, and $(\mathrm{d}, \mathrm{h})$ is the far field diffraction pattern.

can be concluded qualitatively from the video in Figure 2(b). The maximum power coupling efficiency is calculated to be about $22 \%$, and the total efficiency becomes $8 \%$. Again, $14 \%$ efficiency loss is due to the output coupler and the radiation modes in the fiber core. In Figure 5, we show the simulation results with an LMA length of $7.29 \mathrm{~mm}$. Figures 4(a) and 5(e) are the input field intensity and phase distribution, Figures 4(b) and $4(\mathrm{f})$ are the self-imaged field intensity and phase distribution at the input plane, and Figures 4(c) and 4(g) are the field intensity and phase distribution at the partial reflector, respectively. Note that, at the partial reflector the field distribution exhibits a Gaussian like profile. The output far-field pattern intensity and phase distributions are plotted in Figures 5(d) and 5(h), and again the intensity shows a bright central spot.

Similarly, for eight fibers placed in a regular octagon, which has a side length of $32.47 \mu \mathrm{m}$ (four and eight fibers array are placed on the same inscribed circle), the optimum reimaging distance is $14.84 \mathrm{~mm}$, and the LMA fiber length is $7.42 \mathrm{~mm}$, see video inFigure 3(c) for the beam propagation. The power coupling efficiency is numerically found to be about $21 \%$, and the total efficiency is about $7 \%$ which is higher than the 4 fibers case. In Figure 6, we show the simulation results with LMA length of $7.42 \mathrm{~mm}$. For this case, the output near field beam quality is improved; see Figure 6(c). From Figure 6(b), we observe that the central beam spot is brighter than the surrounding beam spots. Although there is no fiber in the center, an additional passive SM fiber with an appropriate mode diameter can be put in the center of the 8 fibers arrays to serve as an output fiber to deliver the Gaussian like beam. This gives an alternative for the design of the laser cavity which will be investigated in the future.

When an additional SM fiber added to the 8 fibers array in the center, the optimum reimaging distance becomes $14.6 \mathrm{~mm}$, and the LMA fiber length is $7.3 \mathrm{~mm}$, see video in Figure 2(d) for the beam propagation. Now the power coupling efficiency increased to $29 \%$ and the total efficiency is increased to $10 \%$. In Figure 6 the simulation results with LMA length of $7.3 \mathrm{~mm}$ display the mode patterns. The result is very close to the 8 fiber array case with even more power concentrated in each SM fiber core as the beam propagates back to the fibers array; see Figure 5(b). Also, the output beam concentrates more power to the central spot in the far-field. 

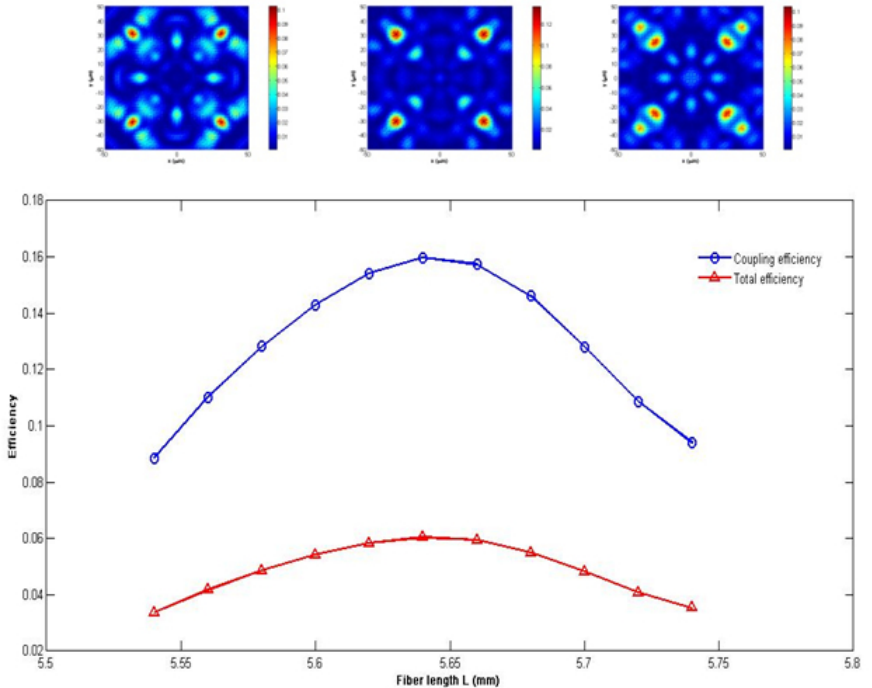

FIG. 7 Power efficiency vs. LMA fiber length. The blue circles are data points for the mode coupling efficiency between the self-imaged beam and the SM fiber array mode, and the red triangles are data points for the total efficiency defined as the ratio of back coupled power to initial power. The lines are guides for the eye. The Top three 2-D images are the intensity distributions of the self-imaged beam for indicated fiber length.

Numerical simulations were also performed by changing the separation between the fibers but keeping them on a regular polygon. The separation parameter changes the self-imaging positions, coupling efficiency, and the output beam profiles. By studying these parameters the coupling efficiency can be optimized.

\section{TALBOT SELF-IMAGING STABILITY}

In the previous section, the numerical simulation results presented used the optimal reimaging distance, which enables the self-imaged beams to couple back into the SM fibers with the maximum overlap to the SM fiber modes. However, the coupling efficiency between the self-imaged beam and the SM fiber modes depends on the LMA fiber length. At the same time, the amplitude and the phase variations among the initial beamlets, and a displacement of the fibers can deteriorate the mode coupling efficiency. In this section, we use the four fibers case to illustrate how the fiber length, phase, amplitude variations, and the fiber displacement affect the mode coupling efficiencies.

\subsection{LMA fiber length variation}

For the 4 fibers case, the optimal LMA fiber length is found to be $5.64 \mathrm{~mm}$ which is half of the reimaging distance. At this fiber length, the self-image can be coupled back into the SM fiber with maximum coupling efficiency which is calculated to be about $16 \%$. Figure 7 shows a the plot of efficiency versus LMA fiber length, where the blue circles show the mode coupling efficiency between the self-image and the SM fiber array mode, and the red triangles show the total efficiency, which is defined as the ratio of the power coupled back to the SM fiber to the initial power. As shown in the figure, the efficiencies drop as the fiber length deviates from the half of

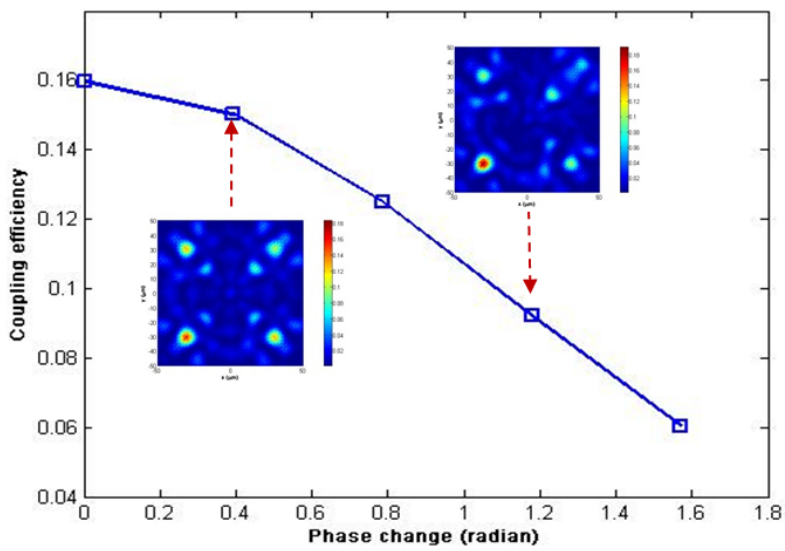

FIG. 8 Coupling efficiency vs. phase mismatch for one fiber. The LMA fiber length is $5.64 \mathrm{~mm}$.

the reimaging distance. The reason is when the fiber length changes, the self-image blurs at the fiber and the SM fiber interface. Thus, the coupling efficiency drops as the fiber length is varied around half the reimaging distance. If $30 \%$ coupling efficiency drop is allowed for the system, then the tolerance of the LMA fiber length would be about $80 \mu \mathrm{m}$. This length requirement is relaxed somewhat if we allow the fiber lasers to tune in frequency.

\subsection{Phase variations}

Through numerical simulation, it is found that the phase variation among the beamlets degrades the coupling efficiency as well. For simplicity, we change the phase of the upper left Gaussian beam as shown in Figure 2(a). In Figure 8, the coupling efficiency vs. the phase change of one fiber is plotted. The coupling efficiency falls as the phase difference increases. The self-imaged intensity distributions that correspond to the phase difference points are also plotted as inserts in Figure 8. It can be seen that the self-images are distorted and blurred, which is also an indication that the coupling efficiency will drop. The reason is the phase difference alters the reimaging distance where the beams interfere with each other, thus the beam can't self-image itself at the SM and LMA fiber interface.

\subsection{Amplitude variations and fiber displacement}

Similar to the change of coupling efficiency due to phase variations discussed in Section 5.2, the amplitude variations will also degrade the coupling efficiency. For a simple example, if the upper right beam spot has amplitude $50 \%$ less than all the other beams, the coupling efficiency is 0.1410 which is a $12 \%$ drop compared with the result fromFigure 3 . This shows that the amplitude variation is not as sensitive as the phase variation. Figure 9 is the plot of the intensity distribution for the initial beam and the beam reflected back to the input plane. $\mathrm{Nu}$ merical simulations also show that when one fiber is displaced by 10 microns horizontally, the coupling efficiency drops $50 \%$ see Figure 10. 

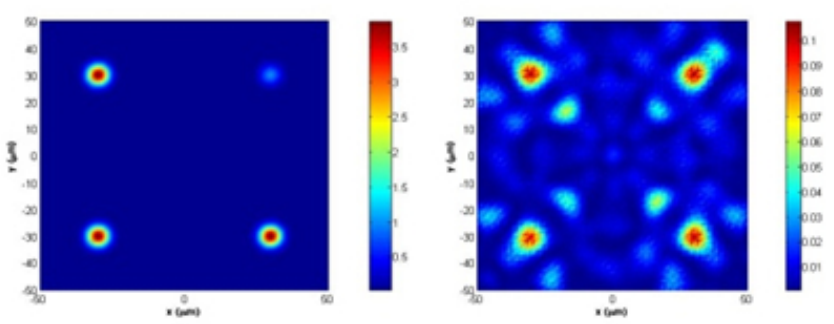

FIC. 9 Self-imaging with intensity variation. (a) is the initial beam intensity distribution, and (b) is the intensity of the beam reflected back to the input plane.
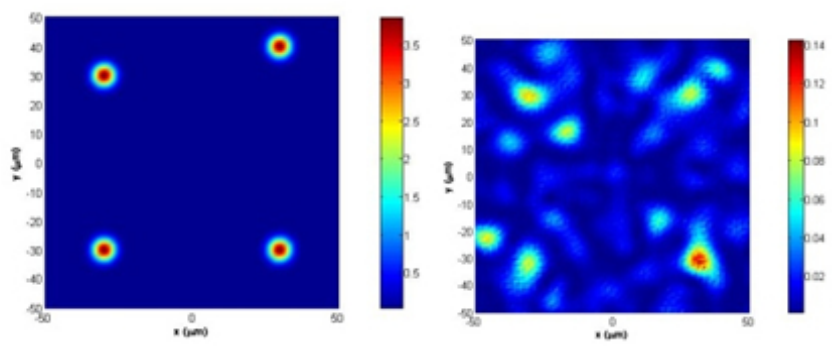

FIG. 10 Self-imaging with fiber displacement. (a) is the initial beam intensity distribution, and (b) is the intensity distribution of the beam reflected back to the input plane.

\section{CONCLUSIONS}

A compact all fiber based phase locking fiber laser design which is capable of phase locking a periodically placed fiber amplifier array is proposed. It is found that adding a fiber in the center increases the coupling efficiency, and the output exhibits a near Gaussian-like profile. The LMA fiber length, amplitude variations among beamlets, and fiber displacement all affect the mode coupling efficiency. The length changes can be managed to optimize the laser performance. Also, it is found that the round trip phase change is sensitive to the input phase of each beamlet and in repeated cavity round trips a single locked phase for all fiber amplifiers will emerge. The reimaging distance is much shorter than expected based on an analysis that uses the fiber core diameter as the periodicity length. Our reimaging length is on the same length scale as reported for annular fibers [24]. This is a clue that the coupled modes with azimuthal angle dependence are important contributors to the reimaging length which is only weakly dependent on the fiber core diameter.

The Talbot mirror fiber device could be pursued as a strategy for scaling up the focusable energy of an array of fiber amplifiers. For instance a hexagonal array of seven fibers could be scaled to a hexagonal array of 19 fibers by adding another periodic ring of fibers. The coupling efficiency increases as the number of fibers is increased and this trend together with a larger periodic array that reinforces the overall periodicity can create more faithful images at the reimaging distance This will be reported in a subsequent publication. The self-organization phasing concept can be a powerful tool in the development of high power multi-fiber lasers.

\section{ACKNOWLEDGEMENT}

RZ was supported by Electro-Optics Program and University of Dayton funding, and he would like to thank his friends in the Electro-optics program for helpful discussions. Dr. Baldemar Ibarra-Escamilla was supported by CONACyT grant 104551.

\section{References}

[1] Y. Jeong, J. Sahu, D. Payne, and J. Nilsson, "Ytterbium-doped largecore fiber laser with $1.36 \mathrm{~kW}$ continuous-wave output power," 0pt. Express 12, 6088 (2004).

[2] A. Galvanauskas, "High Power Fiber Lasers", Opt. Photonics News 15(7), 42-47 (2004).

[3] 0. Schmidt, J. Rothhardt, F. Röser, S. Linke, T. Schreiber, K. Rademaker, J. Limpert, S. Ermeneux, P. Yvernault, F. Salin, and A. Tünnermann, "Millijoule pulse energy Q-switched short-length fiber laser," Opt. Lett. 32, 1551 (2007).

[4] J. Limpert, A. Liem, M. Reich, T. Schreiber, S. Nolte, H. Zellmer, A. Tünnermann, J. Broeng, A. Petersson, and C. Jakobsen, “Lownonlinearity single-transverse-mode ytterbium-doped photonic crystal fiber amplifier," Opt. Express 12, 1313 (2004).

[5] IPG Photonics, Press release, http://www.ipgphotonics.com/ Collateral/Documents/English-US/PR_Final_10kW_SM_laser.pdf

[6] A. E. Siegman, "Propagating modes in gain-guided optical fibers," J. Opt. Soc. Am. A 20, 1617 (2003).

[7] Y. Chen, V. Sudesh, T. McComb, M. C. Richardson, M. Bass, and J. Ballato, "Lasing in a gain-guided index anti-guided fiber," J. Opt. Soc. Am. B 24, 1683 (2007).

[8] A. E. Siegman, Y. Chen, V. Sudesh, M. C. Richardson, M. Bass, P. Foy, W. Hawkins, and J. Ballato, "Confined propagation and near single-mode laser oscillation in a gain-guided, index antiguided optical fiber," Appl. Phys. Lett. 89, 251101 (2006).

[9] V. Sudesh, T. McComb, Y. Chen, M. Bass, M. C. Richardson, J. Ballato, and A. E. Siegman, "Diode-pumped $200 \mu \mathrm{m}$ diameter core, gain-guided, index-antiguided single mode fiber laser," Appl. Phys. B 90, 369 (2008).

[10] T. Her, X. Ao, and L. W. Casperson, "Gain saturation in gain-guided slab waveguides with large-index antiguiding," Opt. Lett 34, 2411 (2009).

[11] H. S. Kim, and M. C. Richardson, "Output characteristics of a gain guided, index anti-guided fiber amplifier under the condition of gain saturation," Opt. Express 18, 15969 (2009).

[12] R. Zhou, B. Ibarra-Escamilla, Q. Zhan, P. E. Powers, and J. W. Haus, "Self-phase modulation effects and pulse propagation in gainguided fibers", J. Europ. Opt. Soc. Rap. Public. 4, 09051 (2009).

[13] Y. Zhou, L. Liu, C. Etson, Y. Abranyos, A. Padilla, and Y. C. Chen, "Phase locking of a two-dimensional laser array by controlling the far-field pattern," Appl. Phys. Lett. 84, 3025 (2004).

[14] B. He, Q. Lou, W. Wang, J. Zhou, Y. Zheng, J. Dong, Y. Wei, and W. Chen, "Experimental demonstration of phase locking of a twodimensional laser arrays using a self-imaging resonator," Appl. Phys. Lett. 92, 251115 (2008).

[15] H. Bruesselbach, D. C. Jones, M. S. Mangir, M. Minden, and J. L. Rogers, "Self-organized coherence in fiber laser arrays," Opt. Lett. 30, 1339 (2005).

[16] L. Liu, Y. Zhou, F. Kong, and Y. C. Chen, "Phase locking in a 
fiber laser array with varying path lengths," Appl. Phys. Lett. 85, 4837-4839 (2004).

[17] J. Cao, Q. Lu, J. Hou, and X. Xu, "Self-organization of arrays of two mutually-injected fiber lasers: theoretical investigation," 0pt. Express 17, 7694 (2009).

[18] L. Li, A. Schülzgen, S. Chen, V. L. Temyanko, J. V. Moloney, and N. Peyghambarian, "Phase locking and in-phase supermode selection in monolithic multicore fiber lasers," Opt. Lett. 31, 2577 (2006).

[19] L. Li, A. Schülzgen, H. Li, V. L. Temyanko, J. V. Moloney, and N. Peyghambarian, "Phase-locked multicore all-fiber lasers: modeling and experimental investigation," J. Opt. Soc. Am. B 24, 1721 (2007).

[20] W. S. Mohammed, A. Mehta, and E. G. Johnson, "Wavelength Tunable Fiber Lens Based on Multimode Interference," J. Lightwave Technol. 22, 469 (2004).
[21] B. Wang, "Review of fabrication techniques for fused fiber components for fiber lasers," Proc. SPIE 7159, 71950A (2009).

[22] C. Pollock, and M. Lipson, Integrated Photonics (Kluwer, Boston, $M A, 2003)$.

[23] C. B, Dane C. B. Dane, L. E. Zapata, W. A. Neuman, M. A. Norton, and L. A. Hackel, "Design and operation of a $150 \mathrm{~W}$ near diffraction-limited laser amplifier with SBS wavefront correction," IEEE J. Quantum Electron. 31, 148 (1995).

[24] M. Hautakorpi and M. Kaivola, "Modal Analysis of the self-imaging phenomenon in optical fibers with an annular core," Appl. Optics 45, 6388 (2006). 